\title{
Evolution of semilocal string networks: Large-scale properties
}

\author{
A. Achúcarro, ${ }^{1,2, *}$ A. Avgoustidis, ${ }^{3, \dagger}$ A. M. M. Leite, ${ }^{4,5}$ A. Lopez-Eiguren, ${ }^{2, \S}$ C. J. A. P. Martins, ${ }^{4,}$ \\ A. S. Nunes, ${ }^{4,6, * *}$ and J. Urrestilla ${ }^{2, \dagger \dagger}$ \\ ${ }^{1}$ Institute Lorentz of Theoretical Physics, University of Leiden, 2333CA Leiden, Netherlands \\ ${ }^{2}$ Department of Theoretical Physics, University of the Basque Country UPV-EHU, 48040 Bilbao, Spain \\ ${ }^{3}$ School of Physics and Astronomy, University of Nottingham, University Park, Nottingham NG7 2RD, \\ United Kingdom \\ ${ }^{4}$ Centro de Astrofísica, Universidade do Porto, Rua das Estrelas, 4150-762 Porto, Portugal \\ ${ }^{5}$ École Polytechnique, 91128 Palaiseau Cedex, France \\ ${ }^{6}$ Faculdade de Ciências da Universidade de Lisboa, Campo Grande, 1749-016 Lisboa, Portugal
}

(Received 7 December 2013; published 5 March 2014)

\begin{abstract}
We report on a detailed numerical study of the evolution of semilocal string networks, based on the largest and most accurate field theory simulations of these objects to date. We focus on the large-scale network properties, confirming earlier indications (coming from smaller simulations) that linear scaling is the attractor solution for the entire parameter space of initial conditions that we are able to probe. We also provide a brief comparison of our numerical results with the predictions of a previously developed onescale model for the overall evolution of these networks. Two subsequent papers will discuss in more detail the analytic modeling of the semilocal segment populations as well as optimized numerical diagnostics.
\end{abstract}

DOI: $10.1103 /$ PhysRevD.89.063503

PACS numbers: 98.80.Cq, 11.27.+d

\section{INTRODUCTION}

The formation of networks of cosmic strings [1-4] is a generic prediction in a wide range of high-energy physics models of the early Universe $[3,5,6]$. Examples include linelike topological defects in field theories breaking $U(1)$ symmetry [7], coherent macroscopic states of fundamental superstrings ( $\mathrm{F}$ strings), and D-branes extended in one macroscopic direction (D strings). The latter two examples, collectively referred to as cosmic superstrings [8], are generically predicted in string theoretic inflationary models involving spacetime-wrapping D-branes [6,9-11]. These "brane inflation" models often have an effective Supergravity description of the hybrid inflation type, ending in a phase transition that produces topological defects, so $\mathrm{F}$ and $\mathrm{D}$ strings can also be modeled as string defects in a field theory approximation.

A key property of cosmic superstrings is that they interact nontrivially, joining together in Y-shaped junctions to form heavier bound FD states $[8,12,13]$, and in this respect, they are similar to non-Abelian strings [3]. There is, however, an important distinction between string defects in ordinary four-dimensional field theories and their higherdimensional superstring cousins: field theoretic strings are known to interact with probabilities of order unity $[14,15]$

\footnotetext{
*achucar@lorentz.leidenuniv.nl

†tavgoust@gmail.com

*up080322016@alunos.fc.up.pt

\$asier.lopez@ehu.es

TCarlos.Martins@astro.up.pt

**0808014@alunos.fc.up.pt

†jon.urrestilla@ehu.es
}

(although at ultrarelativistic speeds, the strings can appear to pass through each other due to multiple intercommutations [16-18]), while cosmic superstrings have intercommutation probabilities which can be much smaller than unity $[19,20]$. This has an important effect on the cosmological evolution of superstring networks leading to higher number densities than for ordinary field theory cosmic strings $[19,21,22]$.

Understanding the evolution of string networks is crucial for predicting their number densities at late times, which in turn determine their potentially observable effects. Since these observational signals depend on parameters of the underlining theory (notably through their sensitivity to the string tensions and intercommutation probabilities), the observational search for cosmic strings provides a powerful tool for probing and/or constraining high-energy physics theories of the early Universe [23-25]. However, the quantitatively accurate modeling of string network evolution is a difficult problem, requiring the combination of a range of techniques (both numerical and analytical) and interpolating between physics at very different energy scales.

For the simplest type of Abelian cosmic strings (e.g., the Nielsen-Olesen solutions of the Abelian Higgs model [7]), which interact simply by exchange of partners, it has long been proposed [26] through analytic modeling that the network should reach a self-similar scaling regime, characterized by a single length scale (the correlation length), which asymptotes to a constant fraction of the horizon. This has been confirmed by high-resolution numerical simulations of both Nambu-Goto [27-29] and field theory [30-33] models, which are in remarkable agreement 
despite the very different techniques employed in each case. Even in this simplest type of strings, there still remains significant numerical uncertainty regarding the relevant importance of decay mechanisms (gravitational radiation vs decay to particles $[32,34]$ ) and the average size of loops in the network [29,35-37], but a consensus has long been reached regarding the large-scale properties of long strings and their quantitative dependence on the intercommutation probability and the rate of cosmic expansion.

The situation is less clear for non-Abelian strings, which do not simply exchange partners but interact in a more complex fashion, forming Y-type junction configurations. This was originally thought to lead to network "frustration," implying a cosmologically catastrophic domination of strings over ordinary matter at late times [38]. Subsequent work, however, has indicated that this is not necessarily the case [39], and whether the network reaches scaling or gets frustrated depends on the relation among the various intercommutation probabilities of strings carrying different charges [40]. In particular, for networks resembling the properties of cosmic superstrings, all recent studies (see, for example, Refs. [40-48]), covering both analytic and field theory modeling, have found scaling solutions with the relative abundance of light $\mathrm{F}$ strings dominating over the heavier D strings and FD bound states. Thus, it is now believed that cosmic superstring networks do reach late-time scaling with light strings being more abundant, even though to date it has not been possible to construct both analytic and field theory models of the "same" network so as to quantitatively compare their abundance predictions.

There is an outstanding case of cosmic string networks for which the cosmological evolution has not been systematically studied: semilocal strings. These are string solutions in theories with both local and global symmetries, the standard semilocal model $[49,50]$ being a minimal extension of the Abelian Higgs model by a global $S U(2)$ symmetry. This model, which has an $S U(2)$ doublet of two equally charged Higgs fields under a single $U(1)$ gauge field, admits stable string solutions even though the vacuum manifold is simply connected. This nontopological nature of semilocal strings endows them with very different properties than their topological counterparts. In particular, they appear as finite open segments for which the ends have long-range interactions akin to global monopoles [51]. Note that such strings are also well motivated from the theoretical point of view, arising in supersymmetric grand unified theories of inflation [52] and the corresponding D3-/D7-brane inflation models [53]. These are a natural extension of usual inflationary models, in which the only extra ingredient is the doubling of a hypermultiplet.

A first study of the cosmological evolution of semilocal strings was presented in Refs. [54] and [55]. The dynamics of these networks is very different than for both Abelian and non-Abelian topological strings, be it global or local. In particular, the long-range forces between the monopoles mean that the segments can shrink and annihilate or grow by joining with other segments. In a recent paper [56], we initiated the analytical study of such networks by modeling them as local strings ending on global monopoles and attempted a preliminary comparison with numerical simulations. Here, we present the first detailed numerical study of semilocal string networks. In this paper (paper I), which is the first in a series of three, we will discuss in detail the large-scale properties of simulated semilocal networks, covering couplings in the range $0.01 \leq \beta \leq 0.09$, and damping terms corresponding to expanding universes dominated by radiation and matter.

Our goal is to demonstrate scaling behavior for semilocal networks. We note, however, that the notion of scaling has to be interpreted carefully in this context. When describing these networks, we may simply be interested in the evolution of the overall energy density contained in the semilocal string configurations, or we may be interested in the detailed distribution of semilocal string segments. The former (which will be the focus of this paper) is the simplest in the sense that it can, to a good approximation, be described by a single length scale, while the latter is somewhat more complex. We will explore this distinction further in the subsequent papers of this series, but for the moment, we emphasize that overall scaling of the network's energy density is necessary but not sufficient to ensure scaling of the segment distribution.

In paper II, we will present the results of detailed comparison of our simulations with the analytical models of Ref. [56]: starting from an initial configuration of a semilocal network, we group all string segments into length bins and evolve the segments in each bin both using our field theory simulation and our analytic models. We then compare the number density in each bin between the two approaches. An important source of uncertainly in this comparison is related to our lack of knowledge of the transverse string velocity in simulations of semilocal strings. In paper III we will present a novel method for measuring velocities from semilocal string simulations.

\section{SEMILOCAL STRINGS}

Semilocal strings [49,50,57] were introduced as a minimal extension of the Abelian Higgs model with two complex scalar fields-instead of just one - that make an $S U$ (2) doublet. This leads to $U(1)$ flux-tube solutions even though the vacuum manifold is simply connected. The strings of this extended model have some similarities with ordinary local $U(1)$ strings, but they are not purely topological and will therefore have different properties. For example, since they are not topological, they need not be closed or infinite and can have ends. These ends are effectively global monopoles with long-range interactions [51] that can make the segments grow or shrink. The 
monopoles at the ends of the strings have some exotic properties by themselves [58].

The relevant action for the simplest semilocal string model, the one we will use in the numerical simulations of Sec. V, reads

$S=\int d^{4} x\left[\left[\left(\partial_{\mu}-i A_{\mu}\right) \Phi\right]^{2}-\frac{1}{4} F^{2}-\frac{\beta}{2}\left(\Phi^{+} \Phi-1\right)^{2}\right]$,

where $\Phi=(\phi, \psi), F^{2}=F_{\mu \nu} F^{\mu \nu}$, and $F_{\mu \nu}=\left(\partial_{\mu} A_{\nu}-\partial_{\nu} A_{\mu}\right)$ is the gauge field strength. It can be easily seen that, setting one of the two scalar fields to zero, we recover the Abelian Higgs model. We can therefore build from the analytical models applied to usual cosmic strings to tackle this new problem.

The symmetry breaking pattern that leads to the formation of strings in this model is $S U(2)_{\text {global }} \times U(1)_{\text {local }} \rightarrow$ $U(1)_{\text {global }}$ so this model can be thought of as a particular limit of the Glashow-Weinberg-Salam electroweak model in which the $S U(2)$ symmetry is global; i.e., the Weinberg angle is $\cos \theta_{W}=0$, and there are no $S U(2)$ gauge fields. The vacuum manifold is the 3 -sphere, so one would not expect strings to form if the dynamics is dominated by the potential energy. On the other hand, the magnetic field is massive, and magnetic flux is conserved, which would suggest the existence of magnetic flux tubes when the magnetic mass is large. This is the regime in which strings form and are stable.

The stability of the strings is not trivial, and it will depend on the value of the parameter $\beta=m_{\text {scalar }}^{2} / m_{\text {gauge }}^{2}$ : for $\beta<1$ the string is stable, for $\beta>1$ it is unstable, and for $\beta=1$ it is neutrally stable $[49,57]$. As we will see in Sec. V, only low values of $\beta$ will be of interest for the comparison because, otherwise, the string network is either unstable or disappears very fast $[54,55,59]$.

After a cosmological phase transition in such a model, it is expected that segments of semilocal strings will form. The cosmological evolution of a semilocal segment network will be quite different from the evolution of ordinary Abelian Higgs strings [50,60]. The fact that semilocal strings have a different cosmological evolution is interesting because cosmic microwave background (CMB) predictions can be different [61] and can be relevant to inflationary model building [52]. Semilocal strings also have interesting gravitational properties $[62,63]$.

The network evolution depends on the interplay between string dynamics and monopole dynamics. When a string segment ends, it must end in a cloud of gradient energy. Those string ends behave like global monopoles, providing an interaction between strings that is independent of distance. Therefore, depending on the interplay between string dynamics and monopole dynamics, the segments can contract and eventually disappear, or they can grow to join a nearby segment and form a very long string, and also the two ends of a segment can join to form a closed loop $[51,54,55]$.
We thus see that, at least to a first approximation, we can envisage these networks as being made of local strings attached to global monopoles, and, as such, previously developed analytic modeling techniques $[64,65]$ should be applicable. This being said, it is also clear that these networks possess additional dynamical properties, beyond those of standard hybrid networks [64-66].

Specifically, the evolution of the string network will depend both on the string tension and on the dynamics of the gradient energy: the latter may be thought of as providing a long-range interaction between the strings. (Note that the force between global monopoles is independent of distance.) In Ref. [56] we presented analytical models for the cosmological evolution of semilocal networks, taking into account these long-range interactions through the addition of phenomenological terms in hybrid (local strings + global monopole) networks. We provide a quick summary of this analytical approach in the next section, before moving to the presentation of our numerical study in Sec. IV.

\section{SEMILOCAL NETWORK EVOLUTION MODELING}

We now discuss an analytic model for the evolution of semilocal string networks, which will be subsequently compared to our numerical simulations. This is mostly a summary of Ref. [56], where the model was first presented; we refer the reader to that work for additional details.

Our analysis focuses on the behavior of the network as a whole, starting from the premise that it can be treated as a network of local strings attached to global monopoles. Therefore, previously developed models for each of these cases can be applied, with suitable changes, to this case. Our model for the evolution of these networks is based on explicitly modeling the dynamics and interactions of the monopoles. This is justified since (as has been shown in previous work [55]) it is indeed the monopoles that control the evolution of the network.

A complementary approach (also developed in Ref. [56]) models the evolution of individual semilocal segments, discussing under what conditions these segments can grow - a process that has been clearly identified in numerical simulations - or shrink. We will not discuss this here since a detailed study of this approach, including comparisons with the numerical simulations discussed in this paper, will be the focus of paper II.

Analytic modeling of defect networks generally starts from the microscopic equations of motion for the defects and uses statistical averaging procedures leading to a macroscopic energy evolution equation (which can be traded for an equation for the network's characteristic length scale) and an equation for the rms network velocity. These equations will necessarily be coupled, and together they describe the network at large scales in a "thermodynamical" sense. Suitable defect interactions are then added 
to these equations in a phenomenological way. This procedure was originally followed in the case of cosmic strings, where it leads to the so-called velocity-dependent one-scale (VOS) model $[64,67]$, which has been well tested against simulations and is used for predicting $\mathrm{CMB}$ signals of string networks [68].

Similar techniques can be used in the case of monopoles. The idea is to obtain an evolution equation for the monopole density (neglecting interactions) and then reexpress it in terms of a characteristic length scale, $L$ (which in this case should be thought of as the average intermonopole distance). The effects of monopole forces and friction are then included in this equation (as well as in the relevant velocity equation) by adding extra phenomenological terms. It has been shown in Refs. $[65,66]$ that the evolution equation for the characteristic monopole length scale has the form

$$
3 \frac{d L}{d t}=3 H L+v^{2} \frac{L}{\ell_{d}}+c_{\star} v
$$

where $c_{\star}$ is a free parameter (to be calibrated by simulations) quantifying energy loss and where we have defined a damping length scale, $l_{d}$, that includes the effects of expansion (due to the Hubble parameter $H$ ) and of friction due to particle scattering (with a generic length scale, $l_{f}$ )

$$
\frac{1}{l_{d}}=H+\frac{1}{l_{f}}
$$

The evolution equation for the rms velocity, $v$, of the monopoles is

$$
\frac{d v}{d t}=\left(1-v^{2}\right)\left[\frac{k_{m}}{L}\left(\frac{L}{d_{H}}\right)^{3 / 2}+\frac{k_{s}}{L} \frac{\eta_{s}^{2}}{\eta_{m}^{2}}-\frac{v}{\ell_{d}}\right],
$$

where the first term in square brackets is the force due to the monopoles

$$
f_{m}=\frac{k_{m}}{L}\left(\frac{L}{d_{H}}\right)^{3 / 2}
$$

and the second describes the force due to the strings,

$$
f_{s}=\frac{k_{s}}{L} \frac{\eta_{s}^{2}}{\eta_{m}^{2}}
$$

For an expansion rate of the generic form

$$
a(t) \propto t^{\lambda}
$$

the Hubble parameter and horizon distance are, respectively,

$$
H=\frac{\lambda}{t}, \quad d_{H}=\frac{t}{1-\lambda} .
$$

The constants $k_{m}$ and $k_{s}$ parametrize the monopole and string forces, and $\eta_{s}, \eta_{m}$ are the relevant symmetry breaking scales. Since in what follows we are mostly interested in latetime scaling solutions, we will (unless otherwise stated) neglect the effect of friction due to particle scattering, which is only relevant in the early stages of the network's evolution.

Note that the fact that the string and monopole symmetry breaking scales appear in Eq. (4) is a consequence of the fact that these equations of motion are obtained by modeling semilocal strings as local strings attached to global monopoles (as previously mentioned) and appropriately adapting the equations of motion for both. Physically one knows that it is the monopoles that dominate the semilocal string dynamics, and this can be modeled by assuming that $\eta_{s} \ll \eta_{m}$. Similarly, the horizon enters in the monopole force term in Eq. (4) due to a number counting argument: this force depends on the number of monopoles (and antimonopoles) inside the horizon; for a detailed discussion, see Ref. [65] and references therein.

We shall mostly consider standard expansion rates, corresponding to the parameter range $0<\lambda<1$, and in particular $\lambda=1 / 2$ in the radiation-dominated era and $\lambda=$ $2 / 3$ in the matter-dominated era. This is justified since observational constraints $[61,68]$ show that semilocal string networks cannot be the dominant component of the Universe's energy budget but can only contribute a small fraction to it.

In the semilocal case, the ratio of the forces due to strings and monopoles is

$$
\frac{f_{s}}{f_{m}}=\frac{k_{s}}{k_{m}}\left(\frac{\eta_{s}}{\eta_{m}}\right)^{2}\left(\frac{d_{H}}{L}\right)^{3 / 2},
$$

and since $\eta_{s} \ll \eta_{m}$ the string force is always subdominant. This is in agreement with theoretical expectations and numerical simulations. Note that this is a distinguishing characteristic of these networks: for local strings attached to local monopoles, the force due to the strings always dominates the dynamics, while for global strings attached to global monopoles the string force is subdominant at string formation but becomes dominant later in the network's evolution [66].

One interesting consequence of the fact that the monopoles always dominate the dynamics is that the only attractor solution of these evolution equations in an expanding Universe (with $a \propto t^{\lambda}$ ) is linear scaling. Indeed, if one looks for generic solutions of the equations of motion for $L$ and $v$, allowing for arbitrary power laws of time in either case, one will find (after a relatively long but otherwise straightforward calculation) that the only consistent asymptotic solution is

$$
L=\gamma t, \quad v=v_{0},
$$

as in the case of plain global monopoles, and indeed the analysis in Ref. [65] is, to a large extent, applicable here. 
There are two possible branches of the scaling solution. First, there is an ultrarelativistic one with

$$
\gamma=\frac{c_{\star}}{3-4 \lambda}, \quad v_{0}=1,
$$

which only exists for slow expansion rates $(\lambda<3 / 4)$ but is in principle allowed both for the radiation and matter eras. Second, a normal solution exists for any expansion rate, with scaling parameters

$$
\begin{gathered}
\gamma=\frac{c_{\star} v_{0}}{3-\lambda\left(3+v_{0}^{2}\right)} \\
\lambda v_{0}=k_{m}(1-\lambda)^{3 / 2} \gamma^{1 / 2},
\end{gathered}
$$

and a constraint on the velocities

$$
v_{0}^{2}<3\left(\frac{1}{\lambda}-1\right) .
$$

This constraint is trivial for $\lambda<3 / 4$ (that is, $v_{0} \rightarrow 1$ is allowed) but restrictive for faster expansion rates. On the other hand, velocities will generically be significant: having $v_{0} \rightarrow 0$ requires $\lambda \rightarrow 1$.

For comparison we also consider the case of Minkowski space (corresponding to $\lambda=0$ and $H=0$ ) but with a friction length scale proportional to the correlation length (say, for simplicity, $\ell_{f} \sim L$ ). This should be an adequate description of some of the numerical simulations of semilocal strings done so far [55]. In this case, linear scaling is still the attractor solution, but the scaling parameters now obey

$$
3 \gamma=v_{0}^{2}+c v_{0}, \quad v_{0}=k_{m} \gamma^{3 / 2} .
$$

In the opposite limit of the fast expansion rate $(\lambda \geq 1$ or, in other words, inflation), the linear scaling solution of Eq. (10) no longer exists. In this case the network is conformally stretched and gradually frozen, and the characteristic length scale and velocity evolve as

$$
L \propto a, \quad v \propto \frac{1}{H L} .
$$

These conformal stretching solutions are ubiquitous in the defects literature.

In the following section, we will test these scaling solutions using state-of-the-art numerical simulations.

\section{NUMERICAL SIMULATIONS}

We simulated numerically the semilocal model introduced in Sec. II so as to provide us with data to be used for comparison with the analytic models mentioned above. The parameter space we want to explore is rather large, so we carefully chose the cases to study and tried to maximize the information we could obtain from our simulations given the computer resources available to us.

We discretized the action given in Eq. (1) by standard techniques (using lattice-link variables and a staggeredleapfrog method) and evolved the discretized action in $1024^{3}$ lattices with periodic boundary conditions, similar to Ref. [55]. One very important approximation we use in our discretization and subsequent evolution of the equations of motion is the use of the so-called fat-string algorithm [69]. We adopt this approach since, otherwise, it would be computationally very expensive to perform the simulations and because it has proven to work fairly well in previous works; in particular, it has been shown that it works fine for obtaining information on large-scale properties, which is our aim in the present work. (A related discussion for the case of cosmic strings can be found in Ref. [70].)

As in many field theoretic simulations of defect dynamics, the initial conditions are an unknown. It would be very hard to simulate exactly the phase transition leading to the formation of the defects, and in many cases, it would not be clear which model to adopt as the underlying phase transition model. However, this is not the goal; instead, our aim is to study the asymptotic (long-term) behavior of these networks and in particular whether (and under what conditions) the expected scaling solution is reached. The art of performing the simulations therefore lies in obtaining some initial conditions which may approach this putative scaling solution as fast as possible. Bear in mind that the periodic boundary conditions force us to have a stringent upper bound on the time that the system can be evolved before it feels the effects of the boundaries. The simulations can only be believed up to the half light-crossing time; i.e., if we sent a light ray in opposite directions in the box, the simulation would be accurate up to when the two rays meet again.

The initial condition chosen for these simulation is the following: the gauge field, gauge field velocities, and scalar field velocities are set to zero. This choice already ensures that Gauss's law is satisfied in the discretized equations and will be satisfied during the dynamical evolution of the system. The scalar fields are chosen to lie in the vacuum manifold but have randomly chosen orientations. After a transient time with an ad hoc damping term for the system to lose energy, the system relaxes into the scaling regime.

Once the system reaches scaling, quantities of interest can be measured. Semilocal strings are not topological entities; therefore, we cannot use topology to detect semilocal strings. For example, in the usual Abelian Higgs strings, one can use the windings of the string to pinpoint where the core of the string is. However, we cannot use the windings in the semilocal strings since the winding is not topologically protected. As mentioned earlier, semilocal strings can be thought of as concentrations of magnetic energy, and that is the strategy we follow, inherited from previous works $[55,71,72]$ : we first calculate the maximum 
of the magnetic field strength, and the radius, of a straight and infinite Abelian Higgs string for a given $\beta$. We use those values for the simulated semilocal string network: if the magnetic field strength of a simulated semilocal model measured at a point of the box exceeds the $25 \%$ of the maximum of the corresponding Abelian Higgs string, we consider that point to be part of a semilocal string segment. The output of our simulation is thus an array of points from the simulated box which have a considerable concentration of magnetic field strength.

One typical simulation snapshot is shown in Fig. 1. It is very clear that semilocal strings have ends (as opposed to Abelian Higgs strings which are either infinite or form


FIG. 1 (color online). Semilocal string network, in matter domination with $\beta=0.04$. The top figure shows two types of structures: on the one hand, we have tubelike structures (proper strings) and on the other short blobs. These blobs we disregard in our analysis. The bottom figure shows the network without blobs, and also each segment has been identified and plotted with a different color. As the number of segments is large, the colors are unfortunately used for more than one string segment. Note also that the blob removal procedure does sometimes fail to identify some spherelike structures since their volume is large. loops). We then group together the points that have been output by the simulations into segments. These segments are mostly tubelike, but some are spherelike instead of tubelike; i.e., they are blobs of energy. These can be formed, for example, after a segment has collapsed into itself. We do not wish to count these blobs as part of our network, and we introduce a lower cutoff: those segments that are not longer than a given factor $(\alpha)$ times the typical radius of a string are considered to be blobs and are discarded. Different choices of $\alpha$ have been considered as explained later. Figure 1 shows the output of a typical simulation where we have differentiated between structures that we consider blobs and proper semilocal string segments. We also show in that figure the network of segments with each segment plotted with a different color.

It is now possible to obtain the necessary quantities for our comparison: the total string length (that is, the sum of all the segment lengths), the number of monopoles, and the segment length distribution. The procedure we have described so far only gives the volume of string points, so in order to obtain string lengths, we divide the number of string points by the typical string width for each $\beta$. The number of monopoles is obtained by multiplying the number of segments by two, as each segment has a monopole and an antimonopole at its ends (more on this point is below). In what follows we do not directly compare the velocities in the model and simulations since reliable numerical measurements of these velocities are highly nontrivial and require the development of additional numerical algorithms, which we will address in paper III. For analogous issues in the more standard case of Abelian Higgs string networks, see Ref. [32]; for the case of domain wall simulations with the Press-Ryden-Spergel (PRS) algorithm, see Ref. [73].

Given a box size (in our case $1024^{3}$ ), one would want to have as big a dynamical range as possible, with as much accuracy as possible. There is clearly tension between these two aims: on the one hand, we would want a big lattice spacing $(\delta x)$ to increase the dynamical range and on the other a small one to increase accuracy in the discretization. We have performed two sets of simulations trying to accommodate both needs: one set of simulations has $\delta x=$ 0.5 and the other $\delta x=1.0$. The first set provides a more accurate discretization of the equations but pays the price of having a shorter dynamical range. The second has a larger dynamical range but may lack accuracy, and there might be discretization effects creeping into the simulation. As will be shown below, results obtained by the two approaches are clearly compatible, and we believe that they are accurate enough for the purposes of this paper.

It is well known [55] that rather low values of $\beta$ are needed to form a reasonably populated network of semilocal strings, and in this work, we chose to perform the simulations for $\beta=0.01, \beta=0.04$, and $\beta=0.09$. The magnetic and scalar string cores for even lower $\beta$ are 
too different in size and are difficult to simulate since they are difficult to resolve and can overlap. Higher $\beta$ gives too scarce a network.

We have performed simulations using two different scale factors ruling the expansion of the Universe, $t^{\lambda}$ : radiation $\left(\mathrm{t}^{1 / 2}\right)$ and matter $\left(\mathrm{t}^{2 / 3}\right)$. Since we are using the fat-string algorithm, this amounts to changing the damping term in each simulation accordingly. All in all, we have performed 12 simulations for each combination of the following parameters:

(i) $\delta x=(0.5,1)$

(ii) $\beta=(0.01,0.04,0.09)$

(iii) Cosmological era $=$ (radiation, matter $)$

There are several systematic errors of which the reader should be aware. On one hand there are numerical errors inherent to the simulation of the dynamics of the system. By these we mean errors arising because of the discretization of the equations (which will depend on the lattice spacing $\delta x$ ), errors coming from the fat-string algorithm, and errors due to the limited dynamical range that can be obtained.

On the other hand, there are systematic errors in the identification and characterization of strings and monopoles from the simulation. The string lengths are obtained by dividing the string volume by the width of a static straight string, whereas our strings can be moving fast (and will therefore have Lorentz contraction) and have turns. In addition, the strings appear more fuzzy depending on the value of $\beta$ and the cosmology (meaning the amount of damping) we use. This fuzziness can sometimes be understood by considering that as strings move there is some radiation left behind, and if such lumps of radiation are touching the string, they are considered as string points by our algorithm. The end result is that the lower the value of $\beta$ and the smaller the damping term, the fuzzier the strings become and also the bigger the energy blobs are.

An illustration of the effect of damping can be found in Fig. 2, where we show snapshots of two simulations, one in the radiation era and the other in the matter era. In radiation, the strings appear to be more fuzzy, with some energy lumps attached to the strings; whereas in the matter era, strings are noticeably smoother. Note that these cubes are only one part of the total simulation, which we have zoomed into to show the fuzziness more clearly; therefore, the segments close to the boundaries would actually be continued in other parts of the box.

As for the effect of $\beta$, for lower $\beta$ the strings are expected to be more stable, as a result of the competition between gradient energy and potential energy [57]. Producing a blob without topology costs the same gradient and magnetic energy regardless of the value of $\beta$, but it does cost less potential energy for smaller $\beta$, thus again producing fuzzier strings.

As mentioned earlier, the number of monopoles is directly read from the number of segments. Some of the


FIG. 2 (color online). Two snapshots of the simulations with $\beta=0.04$ in radiation (top) and matter (bottom). The top figure shows that strings in the radiation era are fuzzier, and many of the segments have energy lumps attached to them. The bottom figure shows segments that are in general smoother-an obvious consequence of the additional damping (there is less radiation in the box). Note that in either snapshot only part of the simulated box is depicted.

segments will in fact form a closed loop, so monopoles would be slightly overcounted by this procedure. Besides, even though we tried to factor out the energy blobs, some of them escaped our algorithm, and we are still counting those blobs as segments and thus overcount monopoles again. Finally, the definition of segment is somewhat arbitrary, since those segments that are not longer than $\alpha$ times the typical radius of a string are discarded. Different choices for $\alpha$ can give different numbers of segments.

Ways of quantifying some of these uncertainties will be briefly discussed in the following section and in more detail in papers II and III. For the moment, we provide one specific example, concerning the choice of the segment cutoff $\alpha$. We have analyzed our data using $\alpha=1,3,5,8$, 
TABLE I. The measured values (with 1- $\sigma$ statistical errors) of the string scaling parameter $\gamma_{s}$ for the various series of simulations described in the text.

\begin{tabular}{lcccccc}
\hline \hline$\beta$ & $\lambda$ & $\delta x$ & $\gamma_{s}(\operatorname{sim})(\alpha=3)$ & $\gamma_{s}($ slope $)(\alpha=3)$ & $\gamma_{s}(\operatorname{sim})(\alpha=8)$ & $\gamma_{s}($ slope $)(\alpha=8)$ \\
\hline 0.01 & Rad & 0.5 & $0.27 \pm 0.02$ & $0.27 \pm 0.04$ & $0.29 \pm 0.02$ & $0.29 \pm 0.04$ \\
0.01 & Rad & 1.0 & $0.280 \pm 0.003$ & $0.27 \pm 0.06$ & $0.285 \pm 0.001$ & $0.29 \pm 0.01$ \\
0.01 & Mat & 0.5 & $0.30 \pm 0.01$ & $0.30 \pm 0.05$ & $0.30 \pm 0.01$ & $0.30 \pm 0.05$ \\
0.01 & Mat & 1.0 & $0.292 \pm 0.002$ & $0.29 \pm 0.01$ & $0.295 \pm 0.002$ & $0.30 \pm 0.01$ \\
0.04 & Rad & 0.5 & $0.301 \pm 0.005$ & $0.30 \pm 0.04$ & $0.294 \pm 0.006$ & $0.30 \pm 0.04$ \\
0.04 & Rad & 1.0 & $0.283 \pm 0.004$ & $0.28 \pm 0.01$ & $0.284 \pm 0.004$ & $0.28 \pm 0.01$ \\
0.04 & Mat & 0.5 & $0.302 \pm 0.001$ & $0.30 \pm 0.03$ & $0.301 \pm 0.001$ & $0.30 \pm 0.03$ \\
0.04 & Mat & 1.0 & $0.291 \pm 0.005$ & $0.29 \pm 0.01$ & $0.291 \pm 0.005$ & $0.29 \pm 0.01$ \\
0.09 & Rad & 0.5 & $0.327 \pm 0.001$ & $0.33 \pm 0.05$ & $0.325 \pm 0.002$ & $0.33 \pm 0.05$ \\
0.09 & Rad & 1.0 & $0.303 \pm 0.005$ & $0.30 \pm 0.01$ & $0.303 \pm 0.005$ & $0.30 \pm 0.01$ \\
0.09 & Mat & 0.5 & $0.337 \pm 0.006$ & $0.33 \pm 0.07$ & $0.336 \pm 0.006$ & $0.33 \pm 0.06$ \\
0.09 & Mat & 1.0 & $0.307 \pm 0.006$ & $0.31 \pm 0.01$ & $0.306 \pm 0.006$ & $0.31 \pm 0.01$ \\
\hline \hline
\end{tabular}

10 , and we have found that the differences are bigger for smaller values of $\alpha$, but for $\alpha=5,8,10$ the results are more consistent. Also, this uncertainty decreases at later times, when there are fewer blobs and strings are longer. Tables I and II, which are described in the next section, show results for different $\alpha$.

Despite these numerical uncertainties, our methodology and sample size are sufficient to establish that the networks reach the expected scaling solution in all the cases studied. We discuss our results and compare them with our analytic models in the next section.

\section{SIMULATION RESULTS AND COMPARISON TO ANALYTIC MODELS}

As described above, we have performed 12 simulations for each case of our set of parameters and used results from the various sets of simulations to obtain basic statistics about the properties of the networks. Each one of the 12 simulations in a given set has the same values for the parameters but a different initial random configuration, so that we can use them to obtain a purely statistical error. All in all, for each one of those simulations, and for specific values of the simulation time, we obtain the total string length $\mathcal{L}(t)$ and monopole number $\mathcal{N}(t)$ in the box. Both of these provide simple diagnostics for the large-scale evolution of the network, and specifically for the presence of scaling, as we will now discuss.

Figures 3 and 4 provide two examples of the evolution of these quantities, for the cases $(\beta=0.09, \delta x=1)$ and $(\beta=0.01, \delta x=0.5)$; both are matter era simulations. These are representative of all the sets of simulations we have performed. This analysis therefore shows that all the networks have reached the scaling solution by the corresponding final time steps. The time needed for the different sets of networks to reach scaling is slightly different, but this is to be expected given the different underlying conditions, such as the amount of damping in the simulation boxes.

The obtained string lengths and number of monopoles can easily be translated into VOS-type length scales using Eq. (10):

TABLE II. The measured values (with $1-\sigma$ statistical errors) of the monopole scaling parameter $\gamma_{m}$ for the various series of simulations described in the text.

\begin{tabular}{lllcccc}
\hline \hline$\beta$ & $\lambda$ & $\delta x$ & $\gamma_{m}(\operatorname{sim})(\alpha=3)$ & $\gamma_{m}(\operatorname{slope})(\alpha=3)$ & $\gamma_{m}(\operatorname{sim})(\alpha=8)$ & $\gamma_{m}($ slope $)(\alpha=8)$ \\
\hline 0.01 & Rad & 0.5 & $0.549 \pm 0.007$ & $0.6 \pm 0.1$ & $0.586 \pm 0.005$ & $0.6 \pm 0.2$ \\
0.01 & Rad & 1.0 & $0.34 \pm 0.01$ & $0.34 \pm 0.02$ & $0.44 \pm 0.01$ & $0.44 \pm 0.03$ \\
0.01 & Mat & 0.5 & $0.544 \pm 0.007$ & $0.55 \pm 0.08$ & $0.555 \pm 0.008$ & $0.56 \pm 0.08$ \\
0.01 & Mat & 1.0 & $0.41 \pm 0.01$ & $0.41 \pm 0.02$ & $0.47 \pm 0.01$ & $0.48 \pm 0.03$ \\
0.04 & Rad & 0.5 & $0.45 \pm 0.02$ & $0.4 \pm 0.1$ & $0.5 \pm 0.2$ & $0.5 \pm 0.1$ \\
0.04 & Rad & 1.0 & $0.359 \pm 0.009$ & $0.36 \pm 0.02$ & $0.469 \pm 0.006$ & $0.46 \pm 0.02$ \\
0.04 & Mat & 0.5 & $0.48 \pm 0.02$ & $0.47 \pm 0.09$ & $0.49 \pm 0.01$ & $0.5 \pm 0.1$ \\
0.04 & Mat & 1.0 & $0.424 \pm 0.006$ & $0.43 \pm 0.02$ & $0.466 \pm 0.004$ & $0.46 \pm 0.02$ \\
0.09 & Rad & 0.5 & $0.45 \pm 0.09$ & $0.45 \pm 0.09$ & $0.46 \pm 0.02$ & $0.5 \pm 0.1$ \\
0.09 & Rad & 1.0 & $0.397 \pm 0.007$ & $0.40 \pm 0.01$ & $0.460 \pm 0.005$ & $0.46 \pm 0.02$ \\
0.09 & Mat & 0.5 & $0.44 \pm 0.01$ & $0.45 \pm 0.06$ & $0.45 \pm 0.02$ & $0.46 \pm 0.08$ \\
0.09 & Mat & 1.0 & $0.419 \pm 0.004$ & $0.42 \pm 0.03$ & $0.442 \pm 0.003$ & $0.44 \pm 0.03$ \\
\hline \hline
\end{tabular}




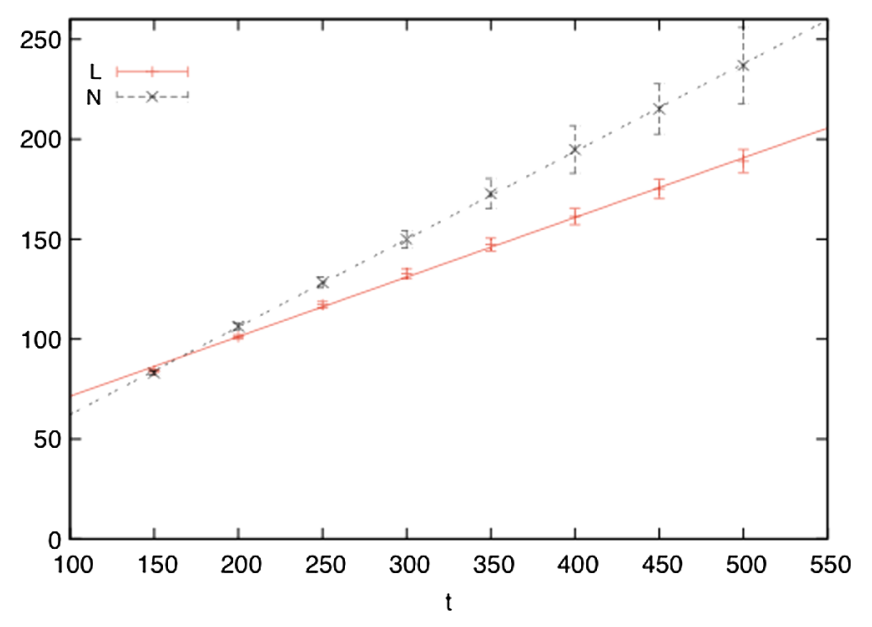

FIG. 3 (color online). Scaling plots for $\mathcal{L}$ and $\mathcal{N}$ for $\beta=0.09$, in the matter era, with $\delta x=1$. The error bars show statistical errors over the 12 simulations.

$$
\begin{gathered}
\gamma_{s} \equiv \frac{L_{s}}{t}=\frac{1}{t} \sqrt{\frac{V}{\mathcal{L}}}, \\
\gamma_{m} \equiv \frac{L_{m}}{t}=\frac{1}{t}\left(\frac{V}{\mathcal{N}}\right)^{1 / 3} .
\end{gathered}
$$

It follows from our discussion in Sec. III that, once a network reaches scaling, both $\gamma_{s}$ and $\gamma_{m}$ should become constants. Note that, while we do expect them to have comparable values of order (but slightly smaller than) unity, there is no expectation that they must be equal.

From our simulations, each of $\gamma_{s}$ and $\gamma_{m}$ can be numerically calculated in various different ways. This turns out to be a simple but useful way of quantifying statistical and systematic uncertainties. We can average the values of $L_{s}(t)$ and $L_{m}(t)$ obtained in each simulation and then calculate $\gamma_{s}$ and $\gamma_{m}$ using Eqs. (17) and (18) on the averaged

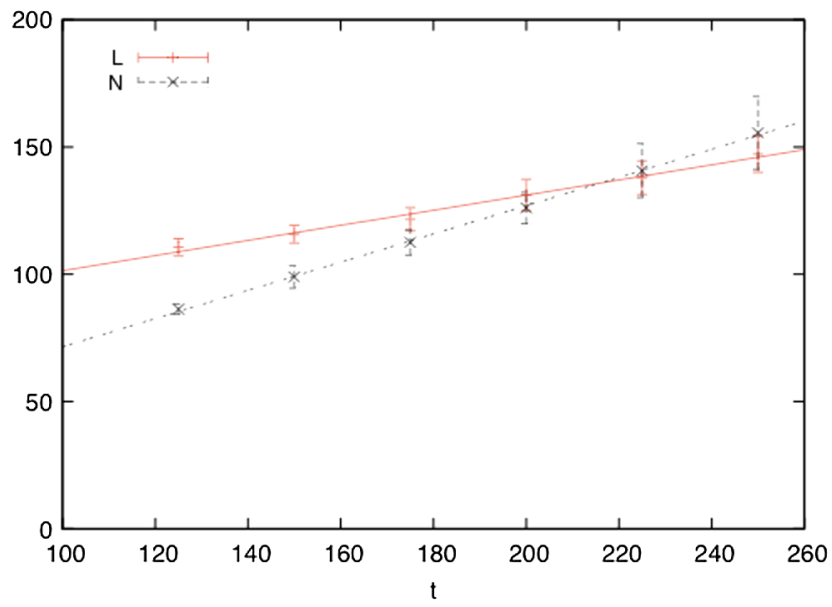

FIG. 4 (color online). Scaling plots for $\mathcal{L}$ and $\mathcal{N}$ for $\beta=0.01$, in the matter era, when $\delta x=0.5$. The error bars show statistical errors over the 12 simulations. quantities, or we can obtain one $\gamma_{s}$ and $\gamma_{m}$ for each simulation and then average the $\gamma_{s}$ and $\gamma_{m}$ over all simulations. Moreover, if we are in (or approaching) scaling, the slopes of $L_{s}(t)$ or $L_{m}(t)$ evolution plots can also be used as numerical diagnostics for the corresponding $\gamma$. This is the prescription we actually use, by considering only the latter part of each set of simulations.

The result of both prescriptions is shown in Table I for $\gamma_{s}$ and Table II for $\gamma_{m}$. The values $\gamma_{s}(\operatorname{sim})$ show the values when the averaging has been done at the simulation level and $\gamma_{m}$ (slope) when it is the $\gamma$ 's which have been averaged. In all cases the errors quoted are statistical errors, which are smaller than the systematic error and so not directly indicative of the full uncertainty. Instead, they should be understood as lower bounds on the uncertainties in these simulations.

Both Tables I and II show comparisons of the simulations with $\delta x=1.0$ and $\delta x=0.5$. As mentioned before, the low value of $\delta x$ is a more accurate approximation to the continuous case but lacks in dynamical range, whereas the higher value of $\delta x$ has a larger dynamical range though a poorer discretization.

The tables also show a comparison of the results obtained with two different values for the definition of segment, namely, for $\alpha=3$ and $\alpha=8$. We investigated values of $\alpha=1,3,5,8,10$ and found that for the latter three the results are quite similar. The table shows that the magnitudes related to the string lengths do not change much with respect to the value of $\alpha$, whereas the monopole length scale changes more. Not only are the cases with a higher value of $\alpha$ more similar to each other, but also the differences between $\delta x=0.5$ and $\delta x=1.0$ are smaller for higher $\alpha$. Therefore, the systematics seem to be under better control for higher values of $\alpha$.

Given the way they were numerically determined, $L_{s}$ should be thought of as the typical interstring distance (or perhaps the typical segment size), while $L_{m}$ is a characteristic intermonopole distance. These are therefore not correlation lengths in the same strict sense as the term is used, for example, in Nambu-Goto string simulations. In particular, the fractal distribution of the semilocal networks (and more specifically the assumption of a Brownian network) is an issue that warrants further study.

Bearing in mind the caveats we discussed, one should proceed with caution if trying to extract quantitative information from these scaling properties. (A further difficulty stems from the fact that we have as yet no accurate measurement of the defect velocities - this will be addressed in paper III.) Nevertheless, it is encouraging that the overall behavior is in agreement with our understanding of the relevant underlying physical mechanisms. Specifically, we note the following:

(i) For a given cosmology (damping term), $\gamma_{s}$ grows with $\beta$, and $\gamma_{m}$ gets smaller. This is to be expected since for lower $\beta$ we expect the system to behave more like an 
Abelian Higgs network, which has longer strings and fewer segments (note that $\gamma_{s}$ and $\gamma_{m}$ are inversely proportional to $\mathcal{L}$ and $\mathcal{N}$, respectively). Analogous results have recently been found for cosmic strings [70].

(ii) For a given $\beta, \gamma_{s}$ is higher for higher damping terms, and $\gamma_{m}$ is lower. This is also to be expected since a lower damping term means that monopole velocities will be higher. Segments can therefore move faster to either grow and meet with other segments or collapse, giving a longer typical string length and smaller number of monopoles. One naturally expects that the additional length lost by segment collapse is more than compensated by that gained by the extra growth. (Note that increasing the string correlation length $L_{s}$ corresponds to decreasing the string density and therefore the total length in string.)

(iii) One set of simulations (corresponding to radiation era, $\delta x=1$ and $\beta=0.01$ ) is an outlier, in the sense that it does not obviously follow the above trends. However, we note that this is the case where there is a smaller effective damping (and therefore more radiation) in the simulation box, and hence this is also the case that is most vulnerable to hidden systematics.

We should also point out that the scaling properties we have obtained for the string segments and monopoles are somewhat less sensitive to the value of $\beta$ than one might have expected. It is possible that this is a feature of the PRS algorithm, as has been recently discussed in Ref. [70]. Nevertheless, our results are consistent with an earlier set of semilocal simulations, discussed in Ref. [56].

As in the case of the analysis in Ref. [56], a full direct calibration of the parameters of the analytic model for the evolution of the overall network cannot be done until we can numerically determine the velocities of the monopoles and segments - a task which we leave for paper III. Still, we can use the results of Table II to provide a preliminary comparison with the model and specifically with the scaling solution described by Eqs. (12) and (13). We will neglect the $\beta$ dependence, which as we saw is numerically found to be quite small when allowing for statistical and systematic uncertainties, and we will focus on the results for the $\alpha=8$ case for the reasons discussed above.

With these assumptions our free parameters are the analytic model parameters, $c_{\star}$ and $k_{m}$, as well as the monopole scaling velocities in the radiation and matter eras, which we will denote $v_{\text {rad }}$ and $v_{\text {mat }}$. Using our numerically determined values of $\gamma_{m}$, we find

$$
\begin{aligned}
v_{\text {rad }} & \sim 0.48 k_{m} \\
v_{\text {mat }} & \sim 0.20 k_{m} ;
\end{aligned}
$$

we have deliberately not included error bars in these numbers since we are unable to quantify possible systematic uncertainties in the $\gamma$ 's. These values are consistent with the results of our earlier simulations [56], where for a faster expansion rate $(\lambda=3 / 4)$ we had found

$$
v_{\text {fast }} \sim 0.12 k_{m} .
$$

As expected, faster expansion rates lead to smaller velocities. On the assumption that the analytic model is correct, we therefore infer that the ratio of the scaling monopole velocities in the matter and radiation eras should be

$$
\frac{v_{\text {mat }}}{v_{\text {rad }}} \sim 0.4
$$

If one assumes a curvature parameter $k_{m}$ of order unity as in the case of Nambu-Goto strings [67], our estimated velocities are comparable to (though possibly somewhat lower than) the ones typically encountered in other field theory defect simulations $[32,73]$. Thus, even though this comparison is somewhat simplistic, the results are at least encouraging. A full comparison (and thus a proper calibration of the analytic model) requires the numerical implementation of a reliable method to measure defect velocities in our simulations, which will be the subject of paper III.

\section{CONCLUSIONS}

We took advantage of recent progress in computing facilities to carry out a more detailed numerical study of the evolution of semilocal string networks, the first results of which have been discussed above. These are based on the largest and most accurate field theory simulations of these objects to date, with sets of $1024^{3}$ simulations. Several of these sets have been simulated, thereby exploring a parameter space spanning different cosmological eras, values of the coupling $\beta$ and spatial resolutions, as well as thresholds for identification of the semilocal segments.

In the present work, we have focused on the large-scale properties of these networks, our main result being a confirmation of earlier indications that linear scaling (analogous to the well-known one for cosmic strings) is the attractor solution for the entire parameter space of initial conditions that we have been able to reliably probe. A brief comparison of our numerical results with the predictions of a previously developed one-scale model for the overall evolution of these networks [56] is encouraging, though a proper comparison must be left for future work. We found the dependence of the scaling parameters on the coupling $\beta$ to be somewhat weaker than one may have naively anticipated. This may be a side effect of our usage of the fat-strings algorithm [69], as recently discussed in a different context in Ref. [70].

As previously mentioned, the dynamics of these networks is more complex than that of plain Nambu-Goto strings, and therefore it cannot be fully described by a 
simple analytic model for the overall defect density. This must be complemented by a description of the evolution of the distribution of the individual semilocal segments. Indeed, the fact that the overall energy density of the network is scaling (which is, physically, what is being quantified by the evolution of $L_{s}$ or $L_{m}$ ) does not by itself ensure that the segment distribution is also scaling. In this sense one can say that a one-scale model is not sufficient to describe the full evolution of the network. To some extent this is analogous to the presence of small-scale structures on cosmic string networks, which can be characterized in Nambu-Goto simulations [29].

The characterization of the semilocal segment population will be the subject of paper II. Indeed, a new way of detecting segments should also be a good way to improve on the possible systematic uncertainties which have been discussed above. Segment identification is clearly the dominant contribution to these, and therefore this is one of the limiting factors preventing a more accurate calibration of the analytic model. The other current bottleneck is a reliable method of measurement of the defect velocities. Note that the two are to some extent related, since velocity measurements will in principle require the direct detection of the positions of the monopoles. Some possible methods to carry out these measurements will be presented and discussed in paper III, leading to a deeper comparison between the analytic models and the numerical simulations and thus to a proper calibration of the models themselves.

\section{ACKNOWLEDGMENTS}

This work was done in the context of Project No. PTDC/ FIS/111725/2009 from FCT, Portugal. The work of A. A. was supported by the Marie Curie Grant No. FP7PEOPLE-2010-IEF-274326 at the University of Nottingham and by a Nottingham Research Fellowship. C. J. M. is supported by an FCT Research Professorship, contract reference IF/00064/2012, funded by FCT/MCTES (Portugal) and POPH/FSE (EC). A. L.-E. and J. U. acknowledge financial support from the University of the Basque Country (EHUA 12/11), Basque Government (IT-559-10), the Spanish Ministry (FPA2009-10612, FPA2012-34456), and the Spanish Consolider-Ingenio 2010 Programme CPAN (CSD2007-00042). A. L.-E. is also supported by the Basque Government Grant No. BFI2012-228. Our numerical simulations were performed on the COSMOS Consortium supercomputer (within the DiRAC Facility, jointly funded by STFC and the Large Facilities Capital Fund of BIS-UK) and on the Milipeia cluster at the Laboratory for Advanced Computing at University of Coimbra.
[1] T. W. B. Kibble, Phys. Rep. 67, 183 (1980).

[2] M. Hindmarsh and T. Kibble, Rep. Prog. Phys. 58, 477 (1995).

[3] A. Vilenkin and E. P.S. Shellard, Cosmic Strings and Other Topological Defects (Cambridge University Press, Cambridge, England, 1994).

[4] E. J. Copeland and T. Kibble, Proc. R. Soc. A 466, 623 (2010).

[5] R. Jeannerot, J. Rocher, and M. Sakellariadou, Phys. Rev. D 68, 103514 (2003).

[6] G. Dvali and S. H. H. Tye, Phys. Lett. B 450, 72 (1999).

[7] H. B. Nielsen and P. Olesen, Nucl. Phys. B61, 45 (1973).

[8] J. Polchinski, arXiv:hep-th/0412244.

[9] C. P. Burgess, M. Majumdar, F. Quevedo, G. Rajesh, and R.-J. Zhang, J. High Energy Phys. 07 (2001) 047.

[10] M. Majumdar and A. Christine-Davis, J. High Energy Phys. 03 (2002) 056.

[11] E. J. Copeland, R. C. Myers, and J. Polchinski, J. High Energy Phys. 06 (2004) 013.

[12] J. H. Schwarz, Phys. Lett. B 360, 13 (1995).

[13] E. Witten, Nucl. Phys. B460, 335 (1996).

[14] E. Shellard, Nucl. Phys. B283, 624 (1987).

[15] R. Matzner and J. Mccracken (1988), http://inspirehep.net/ record/272038.
[16] A. Achucarro and R. de Putter, Phys. Rev. D 74, 121701 (2006).

[17] A. Achucarro and G. Verbiest, Phys. Rev. Lett. 105, 021601 (2010).

[18] G. Verbiest and A. Achucarro, Phys. Rev. D 84, 105036 (2011).

[19] N. T. Jones, H. Stoica, and S. H. H. Tye, Phys. Lett. B 563, 6 (2003).

[20] M. G. Jackson, N. T. Jones, and J. Polchinski, J. High Energy Phys. 10 (2005) 013.

[21] M. Sakellariadou, J. Cosmol. Astropart. Phys. 04 (2005) 003.

[22] A. Avgoustidis and E. Shellard, Phys. Rev. D 73, 041301 (2006).

[23] L. Lorenz, J. Martin, and C. Ringeval, J. Cosmol. Astropart. Phys. 04 (2008) 001.

[24] E. J. Copeland, L. Pogosian, and T. Vachaspati, Classical Quantum Gravity 28, 204009 (2011).

[25] M. Hindmarsh, Prog. Theor. Phys. Suppl. 190, 197 (2011).

[26] T. Kibble, Nucl. Phys. B252, 227 (1985).

[27] D. Bennett and F. Bouchet, Phys. Rev. D 41, 2408 (1990).

[28] B. Allen and E. P. S. Shellard, Phys. Rev. Lett. 64, 119 (1990).

[29] C. J. A. P. Martins and E. P. S. Shellard, Phys. Rev. D 73, 043515 (2006) 
[30] G. Vincent, N. D. Antunes, and M. Hindmarsh, Phys. Rev. Lett. 80, 2277 (1998).

[31] M. Yamaguchi, J. Yokoyama, and M. Kawasaki, Phys. Rev. D 61, 061301 (2000).

[32] J. N. Moore, E. P. S. Shellard, and C. J. A. P. Martins, Phys. Rev. D 65, 023503 (2001).

[33] N. Bevis, M. Hindmarsh, M. Kunz, and J. Urrestilla, Phys. Rev. D 75, 065015 (2007).

[34] M. Hindmarsh, S. Stuckey, and N. Bevis, Phys. Rev. D 79, 123504 (2009).

[35] C. Ringeval, M. Sakellariadou, and F. Bouchet, J. Cosmol. Astropart. Phys. 02 (2007) 023.

[36] K. D. Olum and V. Vanchurin, Phys. Rev. D 75, 063521 (2007).

[37] J. J. Blanco-Pillado, K. D. Olum, and B. Shlaer, Phys. Rev. D 83, 083514 (2011).

[38] D. Spergel and U.-L. Pen, Astrophys. J. 491, L67 (1997).

[39] P. McGraw, Phys. Rev. D 57, 3317 (1998).

[40] A. Avgoustidis and E. Shellard, Phys. Rev. D 78, 103510 (2008).

[41] S.-H. H. Tye, I. Wasserman, and M. Wyman, Phys. Rev. D 71, 103508 (2005).

[42] E. J. Copeland and P. M. Saffin, J. High Energy Phys. 11 (2005) 023.

[43] P. M. Saffin, J. High Energy Phys. 09 (2005) 011.

[44] M. Hindmarsh and P. M. Saffin, J. High Energy Phys. 08 (2006) 066.

[45] J. Urrestilla and A. Vilenkin, J. High Energy Phys. 02 (2008) 037.

[46] M. Sakellariadou and H. Stoica, J. Cosmol. Astropart. Phys. 08 (2008) 038.

[47] A. Avgoustidis and E. J. Copeland, Phys. Rev. D 81, 063517 (2010).

[48] A. Pourtsidou, A. Avgoustidis, E. Copeland, L. Pogosian, and D. Steer, Phys. Rev. D 83, 063525 (2011).

[49] T. Vachaspati and A. Achucarro, Phys. Rev. D 44, 3067 (1991).

[50] A. Achucarro and T. Vachaspati, Phys. Rep. 327, 347 (2000).

[51] M. Hindmarsh, Nucl. Phys. B392, 461 (1993).

[52] J. Urrestilla, A. Achucarro, and A. C. Davis, Phys. Rev. Lett. 92, 251302 (2004).
[53] K. Dasgupta, J. P. Hsu, R. Kallosh, A. D. Linde, and M. Zagermann, J. High Energy Phys. 08 (2004) 030.

[54] A. Achucarro, J. Borrill, and A. R. Liddle, Phys. Rev. Lett. 82, 3742 (1999).

[55] A. Achucarro, P. Salmi, and J. Urrestilla, Phys. Rev. D 75, 121703 (2007).

[56] A. S. Nunes, A. Avgoustidis, C. J. A. P. Martins, and J. Urrestilla, Phys. Rev. D 84, 063504 (2011).

[57] M. Hindmarsh, Phys. Rev. Lett. 68, 1263 (1992).

[58] A. Achucarro and J. Urrestilla, Phys. Rev. Lett. 85, 3091 (2000).

[59] A. Achucarro, J. Borrill, and A. R. Liddle, Phys. Rev. D 57, 3742 (1998).

[60] K. Benson and M. Bucher, Nucl. Phys. B406, 355 (1993).

[61] J. Urrestilla, N. Bevis, M. Hindmarsh, M. Kunz, and A. R. Liddle, J. Cosmol. Astropart. Phys. 07 (2008) 010.

[62] B. Hartmann and J. Urrestilla, J. Phys. Conf. Ser. 229, 012008 (2010).

[63] B. Hartmann, A. Lopez-Eiguren, K. Sousa, and J. Urrestilla, J. High Energy Phys. 03 (2013) 152.

[64] C. J. A. P. Martins and E. P. S. Shellard, Phys. Rev. D 54, 2535 (1996).

[65] C. J. A. P. Martins and A. Achucarro, Phys. Rev. D 78, 083541 (2008).

[66] C. J. A. P. Martins, Phys. Rev. D 80, 083527 (2009).

[67] C. J. A. P. Martins and E. P. S. Shellard, Phys. Rev. D 65, 043514 (2002).

[68] P. Ade et al. (Planck Collaboration), arXiv:1303.5085.

[69] W. H. Press, B. S. Ryden, and D. N. Spergel, Astrophys. J. 347, 590 (1989).

[70] T. Hiramatsu, Y. Sendouda, K. Takahashi, D. Yamauchi, and C.-M. Yoo, Phys. Rev. D 88, 085021 (2013).

[71] J. Urrestilla, A. Achucarro, J. Borrill, and A. R. Liddle, J. High Energy Phys. 08 (2002) 033.

[72] M. Pickles and J. Urrestilla, J. High Energy Phys. 01 (2003) 052.

[73] P. P. Avelino, C. J. A. P. Martins, and J. C. R. E. Oliveira, Phys. Rev. D 72, 083506 (2005). 